\title{
In vitro and in vivo biostability assessment of chronically-implanted Parylene C neural sensors
}

\author{
Aziliz Lecomte $^{\mathrm{a}, *}$, Amélie Degache ${ }^{\mathrm{a}}$, Emeline Descamps ${ }^{\mathrm{a}}$, Lionel Dahan ${ }^{\mathrm{b}}$, Christian Bergaud $^{\mathrm{a}}$ \\ ${ }^{a}$ LAAS-CNRS, Université de Toulouse, CNRS, Toulouse, France \\ ${ }^{b}$ CNRS-UMR 5169, Research Center of Animal Cognition (CRCA), Toulouse, France
}

\begin{abstract}
Parylene $\mathrm{C}$ has rapidly gained attention as a flexible biomaterial for a new generation of chronic neural probes. However, polymeric material failure in the form of delamination, swelling or tearing, often compromises device biostability in the long term. This work constitutes a first step towards lifetime assessment of Parylene C implanted devices. We have conceived a Parylene C-based neural probe with PEDOT-nanostructured gold electrodes for the recording of brain activity. The material response to its biological environment was studied through in vitro soaking tests and in vivo wireless recordings in mice brain, both carried out for up to 6 months. Impedance monitoring and SEM images indicate that over the length of this trial, none of the implants presented with apparent signs of material degradation. Packaging reliability was a predominant factor in device failure, with a certain number of faulty connection appearing over time. This parameter aside, all soaked devices were stable in Artificial Cerebro-Spinal Fluid, with impedances within $10 \%$ of their initial value after 6 months at $37^{\circ} \mathrm{C}$. Besides, at least $70 \%$ of the implanted device were able to accurately record wirelessly high amplitude hippocampal Local Field Potentials from freely-moving mice, with steady Signal-to-Noise Ratio. In other terms, Parylene C implantable sensors responded minimally to artificial and actual physiological conditions during a period of 6 months, which makes them promising candidates for reliable, chronically implanted sensors in the biomedical field.
\end{abstract}

Keywords: Parylene C, PEDOT, chronic neural probes, biostability, in vivo wireless recordings, in vitro soaking

\section{Introduction}

The implantation of neural devices for long periods of time, as in the case of chronic neural interfaces, sets the background to this work. The turn from acute electrophysiology recordings to chronic implantation is a key point for future clinical purposes. To achieve widespread use, the neuroprosthesis must be both innocuous and reliable. These two aspects are highly correlated: the inflammation following device implantation not only constitutes an injury to the brain, but also encapsulates the recording microelectrodes in the form of a glial scar, which eventually results in signal loss over time. Therefore a reduction in inflammatory response has shown to induce greater recording quality and longevity $[1,2]$. The design of a flexible, polymer-based neural probe with a high degree of biocompatibility is meant to alleviate said host response to ensure greater implant biostability [3]. A variety of materials are investigated as substrate for chronic implants, including Polyimide, SU-8, Parylene C, PDMS or more recently, liquid crystal polymers and cellulose nanocomposite, as recently reviewed by Fekete and coworkers [4].

Parylene $\mathrm{C}(\mathrm{PaC})$ has ideal properties as a flexible substrate for chronic neural implantation. Initially used as a coating for implantable electronics, this material rapidly gained popularity as a MEMS material due to the advantages of its deposition process and compatibility with standard micromachining and photolithographic processes. Its pinhole-free polymerization at room temperature and effective gap fill made the coating process compatible with a variety of MEMS materials and structures for applications as

\footnotetext{
*Corresponding author

Email address: alecomte@laas.fr (Aziliz Lecomte)
} 
a coating [5,6] and a structural material [7], although thick Parylene micromachining can be troublesome [8]. Particularly, with an ISO class VI biocompatibility standard and a relative flexibility compared to traditional silicon-based devices (Young's modulus 3.6 GPa), it has become a choice material towards inflammation-reducing chronic neural implant [9].

The biostability of a device refers to its ability to fulfill an intended function in a specific physiological environment as a function of time [10]. Therefore, biostability is a closed-loop reaction between the response of the brain to the implanted object, and the response of said object to the surrounding tissues. In the case of soft polymer-based devices, while the former has been the subject of numerous studies [11, 12, 13,3], the latter remains little known. The mechanisms leading to changes in the properties of biomedical polymers are considered part of biodegradation mechanisms affecting its longevity. In the case of chronically-implanted neuroprosthesis, the implant must be able to record and/or stimulate neural tissues over long periods of time without adversely affecting body fluids, tissues and organs [14]. Prolonged contact in physiological fluids tends to affect the device in the form of electrode corrosion, membrane biofouling, swelling and delamination of the passivation layer [15]. A certain number of in vitro studies focused on the performance of Parylene $\mathrm{C}$ as an encapsulation layer for neuroprosthetic devices. These studies usually include soaking tests, accelerated ageing and/or peeling force measurement to assess adhesion-promoting strategies of Parylene C to a substrate, or its water-barrier capability $[16,17,18,6]$. Though valuable to improve Parylene hermetic properties towards a reliable neural interface, very little studies included the evaluation of Parylene as implant substrate for chronic in vivo purposes, or the appraisal of Parylene-based devices lifetime in the brain. In fact, few studies relate the implantation of Parylene devices and

a

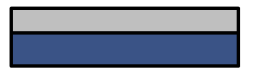

$\mathrm{b}$

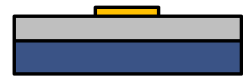

C
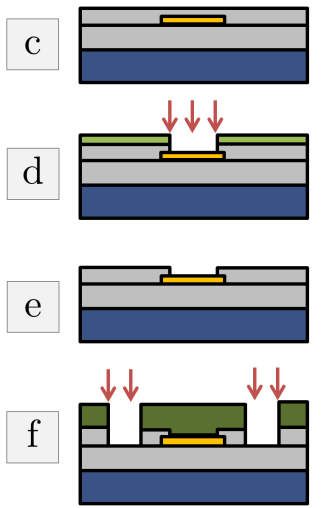

g

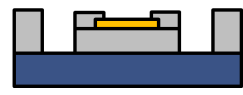

Silicon Parylene Photoresists Metal

Figure 1: Schematic representation of the fabrication process for a Parylene $\mathrm{C}$ probe along with corresponding SEM images.

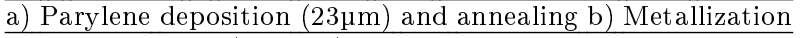
and lift-off of $50 / 200 \mathrm{Ti} / \mathrm{Au}$ metal layer and corresponding SEM image c) Passivation with $1.5 \mu \mathrm{m}$ Parylene $\mathrm{C}$ and annealing d) Electrode opening via passivation plasma etching e) Photoresist stripping and corresponding SEM image f) Plasma etching through the whole thickness for device release g) Photoresist stripping and corresponding SEM image. All parameters and references for this fabrication process are available as a Supplementary Data. monitoring of brain activity over long period of time. Some involve short-term implantation, between 3 weeks [19] and 5 weeks [20, 21]. Recently, studies by Ellis Meng and coworkers showed proof of electrical recordings in rats for a period of 3 to 12 months, and primary investigation was carried out over signal quality [11], as well as immunohistochemical analysis [12]. But overall, if Parylene $\mathrm{C}$ is largely being investigated as a substrate for chronically-implanted neural probes, the stability of Parylene-based devices lacks crucial perspective. In order to achieve such hindsight, in vitro appropriate models and in vivo accurate experiments need to be intensely pursued.

This study intends on focusing on material alteration of Parylene $\mathrm{C}$ devices over the long term. We have conceived a Parylene C-based neuroprosthetic device, with gold patterns as recording microelectrodes. These electrodes are nanostructured via electrodeposition of poly(3,4-ethylenedioxythiophene) (PEDOT). This conductive polymer benefits from a high conductivity, biocompatibility and excellent stability, which favors recording capability for neural applications $[22,23,24]$. In this study, we intend to show a preliminary evidence of the biostability of our Parylene implant for chronic neural applications, through in vitro soaking tests in artificial brain environment and prolonged in vivo wireless recordings in mouse brain for up to six months. 


\section{Materials and methods}

\subsection{Parylene $C$ neural probe}

In previous studies, we demonstrated the fabrication and functionality of a Parylene C-based neural probe $[25,26]$. The fabrication process is shown on Figure 1 and described more precisely in the Supplementary Data information. Briefly, the implant consists of gold patterns sandwiched between two Parylene C layers, respectively 23 and $1.5 \mu \mathrm{m}$ thick. The devices are annealed during 16 hours at $100^{\circ} \mathrm{C}$ under nitrogen flow, to increase the adhesion between the Parylene layers. Finally, to cut through this 25um-thick implant, deep plasma etching was optimized [27], and the resulting unitary devices can be easily peeled off with tweezers. To fit with the in vivo wireless recording system, each device contains four recording microelectrodes (or simply "electrodes") of $40 \mu \mathrm{m}$ in diameter, patterned on a short $2 \mathrm{~mm}$-long, $250 \mu \mathrm{m}$-wide probe (Figure 2). The electrodes are then nanostructured via electrochemistry through a potentiodynamic route from a PEDOT:PSS solution [25]. To prevent the device from buckling during implantation, the shank is backed with a resorbable silk fibroin coating following a procedure optimized elsewhere [26]. The silk fibroin film is gutter-shaped, with maximal dimension of about $130 \mu \mathrm{m}$, and acts as a stiff support that enables insertion in brain tissues. Depending on the application, silk fibroin degradation rate can be tunable depending on a shear treatment (water-annealing) that modifies the inherent fibroin structure [28]. Here, because the surgical procedure requires fast positioning, the silk coating is not water-annealed, therefore allowing the silk to resorb within a few hours or days in the tissues.

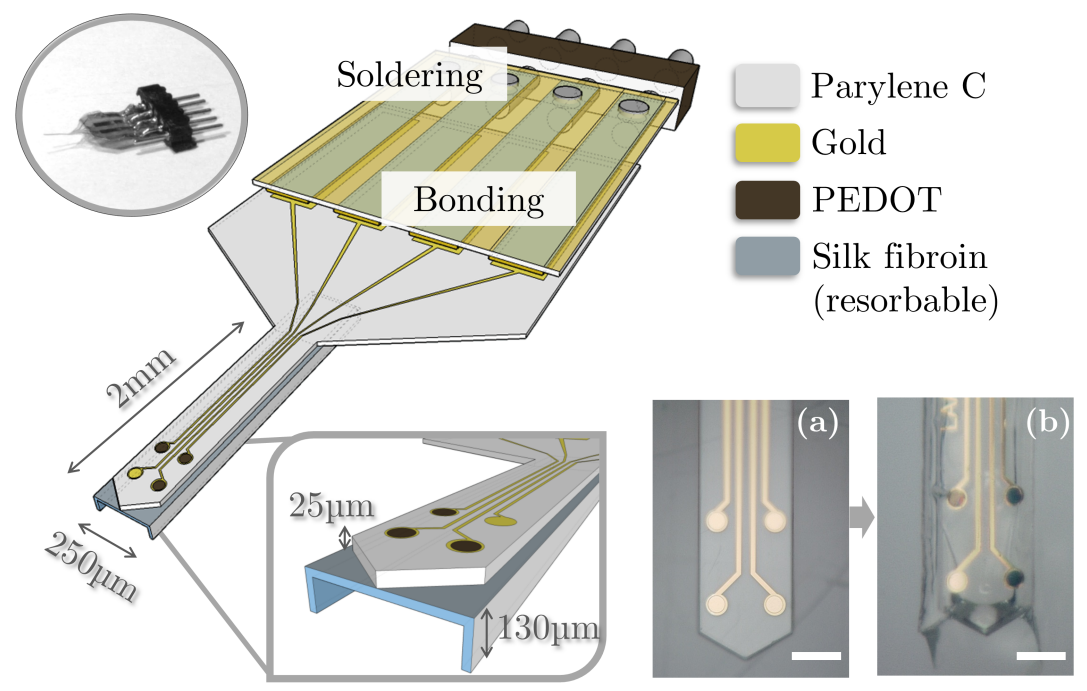

Figure 2: Schematic representation (not to scale) of a Parylene C-based neural probe with three PEDOT-nanostructured electrodes (40 $\mathrm{mm}$ in diameter) and one gold electrode as control. Insertion is assisted with a bioresorbable silk fibroin coating on the backside of the shank. The packaging involves the bonding of the device onto a flexible polyimide cable with Anisotroptic Conductive Film, and soldering of the latter onto a pin connector adapted to the mouse wireless acquisition system. Two photographs show the probe tip (a) before and (b) after PEDOT nanostructuration and silk integration (scale bar $150 \mu \mathrm{m})$.

\subsection{Packaging}

The probe is bonded on a customized flexible polyimide cable with golden traces (or "flex", from Shenzhen Sienta Industry Co) via Anisotropic Conductive Film (ACF 7371-20, 3M) (Figure 2). ACF is an adhesive containing metal beads that form a unidimensional contact when pressed and heated, ensuring a strong, reliable electrical connection without cross-over between the contact pads. Bonding was performed using a TTB-1008 bonder from ITO-GROUP and the pressure/temperature parameters were optimized. First, ACF is pre-tacked onto the polyimide flex at $0.05 \mathrm{MPa}$ and $80^{\circ} \mathrm{C}$ (setpoint temperature $130^{\circ} \mathrm{C}$ ) during 5 seconds. Then the Parylene device is bonded to the flex at the same pressure at $170^{\circ} \mathrm{C}$ (setpoint temperature $260^{\circ} \mathrm{C}$ ) during 20 seconds. The overall connection system is then passivated with an insulating biocompatible glue (Polytec EP 601). For in vitro testing, this long flex is easily plugged 
into a ZIF connector. For the in vivo procedure, the flex is pierced and soldered at $400^{\circ} \mathrm{C}$ using a lead-free soldering wire onto a $1.27 \mathrm{~mm}$-pitch pin connector (DigiKey) fitting the commercial wireless acquisition system. This step occurs before the probe is bonded onto the flex, because Parylene would not withstand such high soldering temperatures.

\subsection{In vitro soaking tests}

Soaking tests are often carried out to evaluate implants degradation over time [6]. The choice of soaking conditions is essential to reproduce the in vivo environment. Usually, the liquid consists of PBS or $\mathrm{NaCl}$ at $0.9 \% \mathrm{w} / \mathrm{v}$, that mimics general physiological conditions. Accelerated ageing tests, where the medium is brought to $60^{\circ} \mathrm{C}$ and/or with ultrasonic stress, is a way to perform these tests [17, 16]. Here, we choose a more elaborate medium, the Artificial Cerebro-Spinal Fluid (ACSF), that resembles ionic concentrations and $\mathrm{pH}$ in brain environment (Table 1), and we consider a more biomimicking approach for the soaking conditions, so that all samples are soaked at $37^{\circ} \mathrm{C}$ in an oven during six months. Four Parylene implants with each four PEDOT-modified gold electrodes are immersed into the same volume of ACSF (25mL) in plastic cups, with the lid sealed to prevent water from evaporating (Figure 4A). Every week or few weeks, the samples are retrieved from the oven and let cool at room temperature until they reach $20^{\circ} \mathrm{C}$. Electrochemical Impedance Spectroscopy (EIS, $10 \mathrm{~Hz}-10 \mathrm{kHz}$ ) is then performed at room temperature on those electrodes directly in the soaking medium, with a platinum wire as counter and reference electrode. ACSF contains $0.725 \% \mathrm{w} / \mathrm{v} \mathrm{NaCl}$ along with other ions in solution (Table 1), and preliminary results showed that the difference between EIS measurement in $\mathrm{NaCl}$ or in ACSF solutions is slight, in the order of magnitude of EIS variability measurement (Impedance and phase data are available as supplementary data). SEM images are taken at different times to evaluate morphology evolution and the EIS is carried out directly in the soaking medium,

\begin{tabular}{|c|c|c|c|c|c|c|c|}
\hline Solution & $\mathrm{NaCl}$ & $\mathrm{NaHCO}_{3}$ & $\mathrm{KCl}$ & $\mathrm{MgSO}_{4}$ & $\mathrm{NaH}_{2} \mathrm{PO}_{4}$ & $\mathrm{CaCl}_{2}$ & Glucose \\
\hline \hline Concentration (w/v) & 0.725 & 0.218 & 0.026 & 0.012 & 0.015 & 0.013 & 0.018 \\
\hline
\end{tabular}

Table 1: ACSF composition used in this study

\subsection{In vivo chronic recordings}

To complete this in vitro soaking step, a preliminary chronic in vivo trial is carried out. Five Parylene probes containing both PEDOT and gold electrodes are implanted in five mouse hippocampi for a duration of 11 to 29 weeks. Neural signals are recorded regularly during the trial length, and impedance and morphology variations before and after implantation are assessed. Because extracting the probes after implantation would compromise the integrity of the brain, we were not able to perform immunohistochemical analysis of the glial scar, and favored the analysis of material reaction to long-term implantation.

Implantation. Five C57BL/6 mice (Charles River) are anesthetized with isofluorane (Vetflurane) and set to a stereotaxic apparatus. After a first skin incision, a craniotomy is performed, and the dura mater of the meninges is incised to allow access to the pia mater. Thanks to the presence of silk on the backside, each Parylene shank is successfully inserted in the CA1 region of the hippocampus with an automatic micropositioner (LN Junior22, Luigs \& Neumann) at a relatively high speed of $150 \mathrm{pm} / \mathrm{sec}$, and a first level of dental cement is mounted on the shank base to fix its position. The tip of the probe is placed at the following coordinates: $1.8 \mathrm{~mm}$ posterior and $1.3 \mathrm{~mm}$ lateral from bregma and $1.6 \mathrm{~mm}$ ventral from brain surface. The reference electrode (microscrew with silver wire) is screwed to the occipital bone. Finally, the whole apparatus is fixed in a second layer of red dental cement, so that only the connector pins are accessible on top of the mouse head. The skin is then sutured around the dental cement.

Recordings. To let the mice recover, the wireless recording sessions start after one week post-implantation. During these five-minutes recording sessions, each mouse is connected to a headstage (MultiChannel System W4-HS), mounted with a wireless battery on top (MultiChannel System W2100-B-30mAh-BB) and transferred to the recording cage. As the animal is discovering this unknown environment, its brain activity is recorded using the MultiChannel System data acquisition system (Figure 3a). After five 
minutes, the headstage is removed and the animal placed back in its cage. The experiment is then carried out on the following mouse.

Data treatment. Hippocampal local field potentials (LFP) are recorded without amplification or filtering using the MultiChannel System acquisition software. The hippocampus is a region of brain involved in both memory consolidation and spatial navigation. In rodents, spatial navigation leads to a very characteristic brain signal composed of Theta oscillations at around 7-12 $\mathrm{Hz}$ (Figure 3b), where smaller and faster Gamma oscillations are nested $(40-80 \mathrm{~Hz})[29,30]$.

To determine whether an electrode is in working conditions, that is to say able to accurately record these oscillations, the spectrum analysis of these unfiltered LFP is used. The spectrum analysis is obtained with Spike2 software (CED System), with a Hanning window function and a $0.61 \mathrm{~Hz}$ resolution. As signal amplitude is highly dependent on animal behavior, this analysis was performed between minute 3 and minute 5 of the recording sessions.

To calculate the Signal-to-Noise Ratio (SNR) of the functional electrodes, we use the definition below:

$$
S N R=\frac{A}{2 \times S D_{\text {noise }}}
$$

where $A$ is the amplitude of the signal, that is the peak-to-peak voltage of the mean waveform, and $S D_{\text {noise }}$ is the standard deviation of the noise [31]. The recorded signal is smoothed using the Smooth Data Processing tool in Spike2 software (low-pass filter at $200 \mathrm{~Hz}$ cut-off) and the noise is considered as the difference between the raw signal and the smoothed signal. It is important to stress out that this definition of the SNR is usually applied in electrophysiology to define the quality of spike sorting [32, 24], where the amplitude of the signal is independent from experiment condition, since a spike amplitude is constant. In this case, the SNR is hard to define, because depending on experiment conditions (animal behavior, implant position, etc.) the signal amplitude varies independently from electrode quality. Therefore, assessing the evolution of device electrical capacity over time is troublesome. Here, for each electrode in working condition the SNR is calculated at 5 different intervals of 5 seconds from each recording sessions.

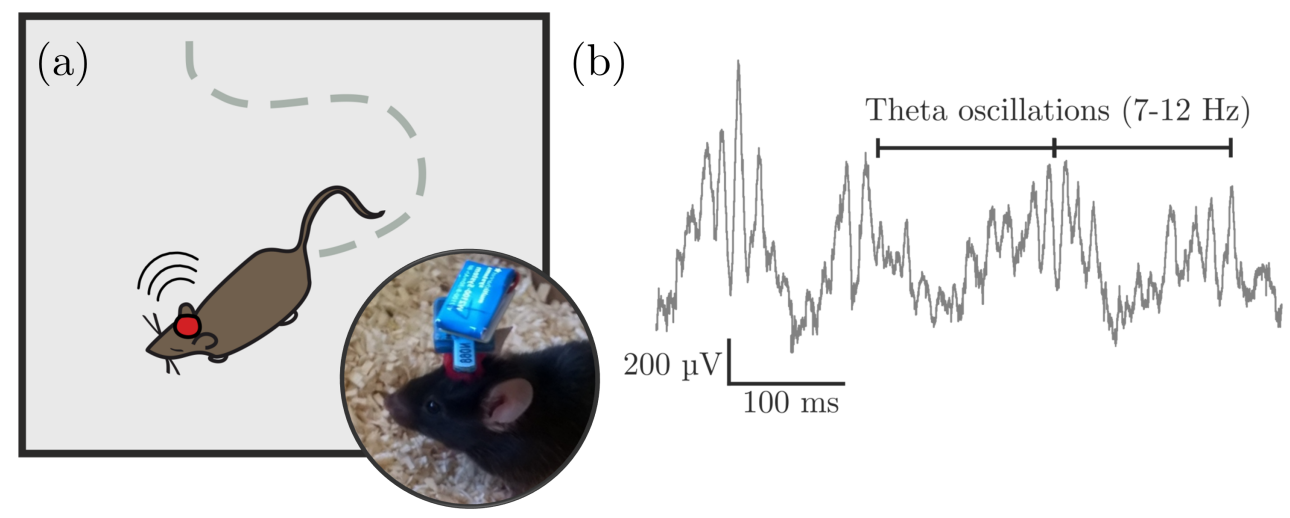

Figure 3: (a) Schematic representation and photograph of an in vivo wireless recording session. A freely-moving mouse is actively exploring an unknown environment, and its hippocampal activity is recorded wirelessly with a Parylene-based neural probe connected to a commercial headstage and battery (in blue). (b) Typical hippocampal activity displayed during a recording session. Spatial navigation of the animal induces large Theta oscillations at around 7-12 Hz.

\section{Results and discussion}

\subsection{Evolution of Parylene implants in brain-resembling environment}

Throughout the 29-weeks study, the impedance of the 16 PEDOT-modified electrodes remained considerably stable, as depicted on Figure 4B. To simplify the comparison, this box plot displays the impedance magnitude of each electrode at $1 \mathrm{kHz}$. Indeed, although slow Local Field Potentials (0-100 $\mathrm{Hz}$ ) were recorded afterward, impedances at $1 \mathrm{kHz}$ are used in this study as conventionally done in electrophysiology because it corresponds to a spike timescale. The impedance median measured over 
A
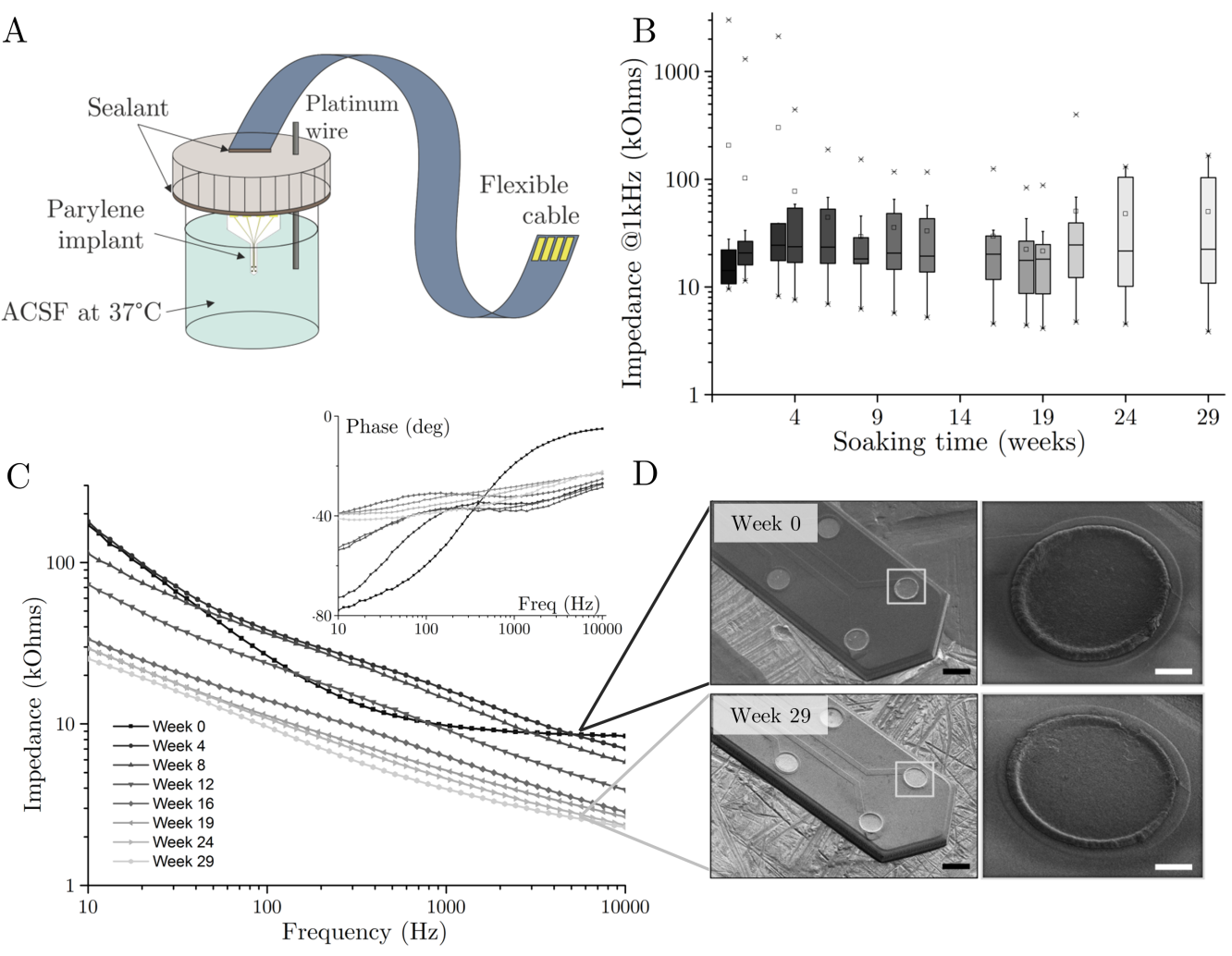

Figure 4: A. Schematic representation of the soaking test experimental setup. Each Parylene probe is mounted on a flexible cable and immersed into $25 \mathrm{~mL}$ ACSF at $37^{\circ} \mathrm{C}$ during a period of six months. Electrode impedance is monitored using a platinum wire as a counter and reference electrode. B. Standard box-and-whisker plot representing the evolution of impedance at $1 \mathrm{kHz}$ for these 16 PEDOT-nanostructured soaked electrodes (diameter $40 \mu \mathrm{m}$ ) as a function of time. The filled rectangle is the interquartile range (IQR $=\mathrm{Q} 3-\mathrm{Q} 1)$, with the mean $(\square)$ and median $(-)$ values of all impedances. Whiskers are $1.5 \times$ IQR and outliers are represented as *. C. Impedance magnitude and phase example for one PEDOT-modified electrode. In this case, the impedance magnitude tends to slightly decrease over time. D. SEM images of this implant before immersion and after 29 weeks soaking in ACSF (black scale bar is $50 \mu \mathrm{m}$, white scale bar is $10 \mu \mathrm{m}$ ). The Parylene devices show no significant sign of delamination or swelling at the microscopic level, and neither do the PEDOT-nanostructured electrodes, which corroborates their impedance stability.

the course of the 6-months trial lies between 10 and $25 \mathrm{k} \Omega$ at $1 \mathrm{kHz}$, and $90 \%$ of all impedance values are below $100 \mathrm{k} \Omega$ at all times, which are rather suitable values for high-resolution electrophysiological recordings [26]. Besides, most outliers are one order of magnitude above this impedance median, and result most likely from flawed electrodes after Clean Room fabrication (problems in passivation etching, for instance) or faulty contacts or packaging. Indeed, one of the four implants showed early signs of unsteady water sealing, resulting in poor electrical capability. On the other hand, the impedance of all electrodes on the three other implants were remarkably stable, that is to say within $10 \%$ of their initial value. Some impedances even slightly decreased (Figure 4C), which we first thought to come from a slight change in the composition of the ACSF medium (evaporation, ion precipitation), but a renewal of the medium at around week 19 did not seem to alter impedance measurement. It could have also come as a consequence of the beginning of delamination of Parylene around the electrode, resulting in a subtle increase of electrode diameter thus lower impedance magnitude, but a closer look at the morphology of the device seems to refute this hypothesis.

Indeed, SEM images were carried out on all soaked implants before and after their immersion in ACSF. Over the four devices, none showed significant signs of Parylene delamination or swelling (Figure 4D). The passivation layer remains well-retained on the surface, and the device sides appear unaltered, besides the presence of a few aggregates from ion precipitation on the Parylene surface. From a microscopic view, the PEDOT coatings seem unchanged after such long immersion in liquid. Therefore, the slight decrease in impedance magnitude observed for several electrodes is more likely a product of PEDOT slight swelling 
over time, with stretched polymer chains inducing greater charge transfer. Besides, the dissolution of potential residues on the surface in the medium could also contribute to impedance magnitude decrease and positive shift in impedance phase over the first week of study.

In conclusion, the four implants seem to withstand surprisingly well prolonged contact in liquid environment. In the light of the first outcome, no significant sign of alteration of neither the Parylene substrate nor the PEDOT nanostructuration were observed after 6-month immersion, correlated with stable impedance measurement over time. Soaking for longer periods of time, or with increased temperature, should allow us in the future to assess the mechanical holding of our Parylene devices for chronic implantation.

\subsection{Stability of Parylene implants in vivo}

\subsubsection{Evolution of functional electrodes over time}
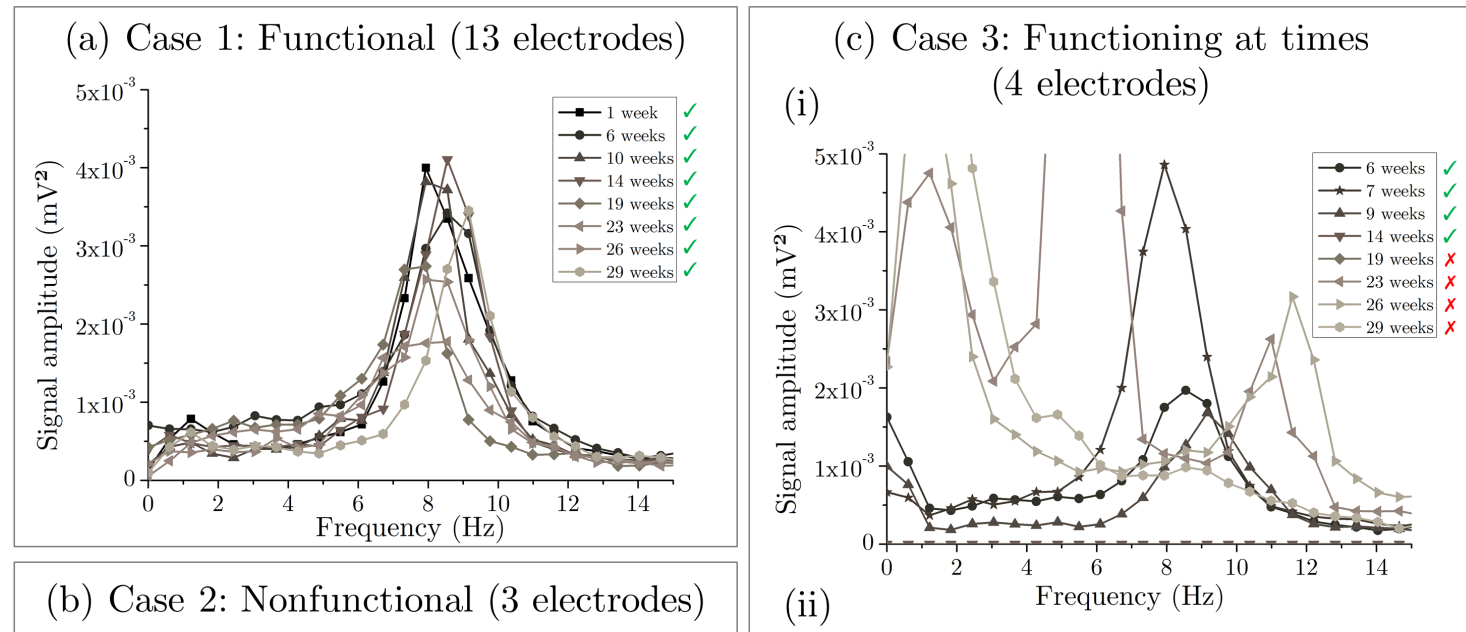

(b) Case 2: Nonfunctional (3 electrodes)
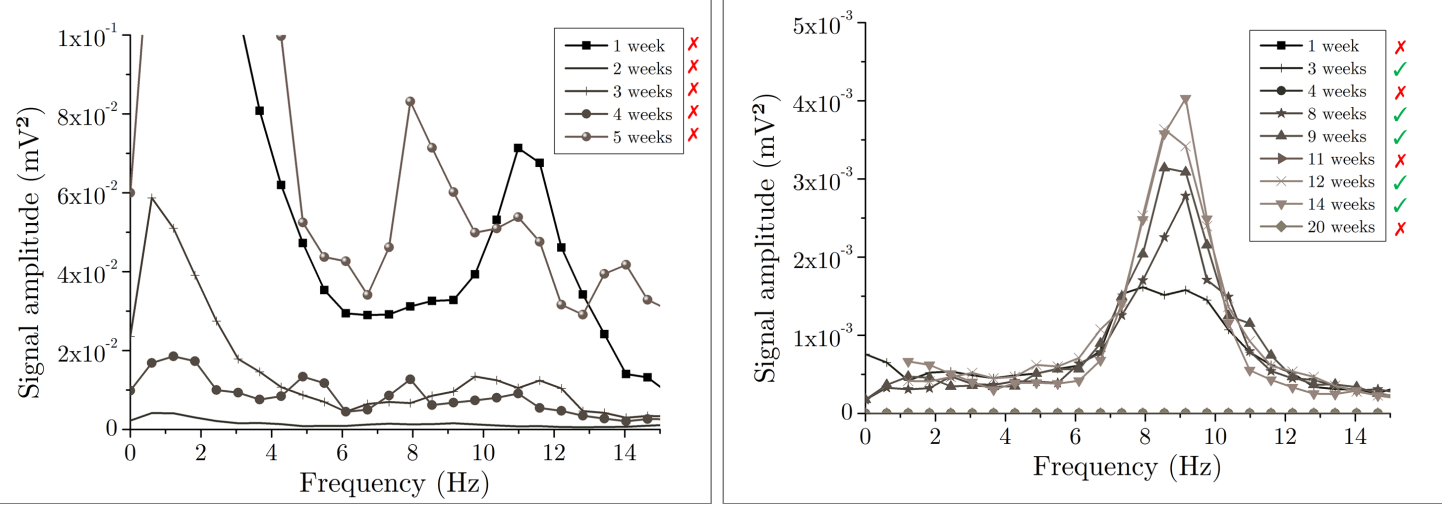

Figure 5: Examples of spectral representation of the signal recorded during 120 seconds on different electrodes. (a) Case 1: 13 electrodes were functioning during the whole procedure. The stable signal displays a noticeable Theta oscillation $(7-12 \mathrm{~Hz})$ with rather stable amplitude. (b) Case 2: 3 electrodes were unable to record hippocampal activity. The signal is uncertain from the first recording session, consistent with either short circuits or mechanical damage of the probe during insertion. (c) Case 3: 4 electrodes were at times either functional or nonfunctional. (i) After approximately 14 weeks $i n$ vivo, the unstable electrode has lost the ability to accurately record brain activity: the signal is displays a variety of power amplitudes in the spectral representation. (ii) At given times, the electrode displays either stable or unstable amplitude. Note that on a given device implanted in a given mouse, both functional and nonfunctional electrodes coexisted. Therefore, as the glial scar would be affecting electrical capability of all the electrodes on one device, the unability to record appropriate LFP is more likely a cause of specific electrode damage or faulty contacts.

A functional microelectrode is defined as an electrode that is able to accurately record hippocampal oscillations typical of a mouse actively discovering an unknown environment. It is relatively easy to determine whether an electrode is in working conditions by analyzing the spectral representation of a recording session, as depicted on Figure 5. As mentioned earlier, the power spectrum analysis is given 
for the last two minutes of each recording sessions to clear the signal from the unpredictability of animal behavior. This analysis enables us to classify all electrodes implanted (4 electrodes per device, 5 devices) into functioning categories. It is important no note that two mice presented with medical issues and had to be sacrificed after only 8 weeks of implantation. Overall, the number of functional recording electrodes in vivo in this trial varies between 70 and $90 \%$, depending on the recording session.

Case 1: A functional electrode displays a high and stable amplitude Theta component around 7-12 $\underline{\mathrm{Hz}}$ (Figure 5a) throughout the recording sessions. In this experiment, 13 electrodes were in working conditions the whole implantation time.

Case 2: Three electrodes on three separate devices were not operating from the first recording session (Figure 5b). The signal is uncertain from week 1, with either amplitudes reaching over 20 times that of the stable signal presented in 5a, or zero amplitude. This issue is consistent with either short circuits or mechanical damage of the probe during insertion.

Case 3: During the length of the trial, four electrodes on three separate devices presented with sporadically or long-term unstable spectral representation. For instance, at a certain time, the signal turns from a distinctive Theta component to a lack of oscillation, as seen on Figure 5c-i. For other electrodes, the signal is lost in some recording sessions, but is back on the next one.

The foreign body response has often shown to cause glial scarring and device migration that results in signal loss. However, it is reasonable to assume that such body reaction would affect a device as a whole. In this experiment, both functional and nonfunctional/malfunctioning electrodes were present on all of the devices. For instance, the signals displayed in Case 1 and Case 3i in Figure 5 are coming from two electrodes at the same insertion depth over the same device, at a distance of 100 $\mathrm{mm}$. Therefore, the most legitimate assumption would be that signal loss is mainly due to faulty contacts and short circuits, or electrode mechanical damage. Indeed, the constant animal movements are likely to cause stress on the connectors over time.

\subsubsection{Evolution of signal quality}

The evolution of signal quality gives partial information on how the devices withstand their new environment. The Signal-to-Noise Ratio (SNR) represents a ratio between the meaningful signal and the measurement noise, and its calculation is described in the experimental section. Here, we focus on the recording electrodes that were considered functioning in relation to the criteria presented above (Case 1, $\underline{13 \text { electrodes) }}$ and the results are plotted on Figure 6.

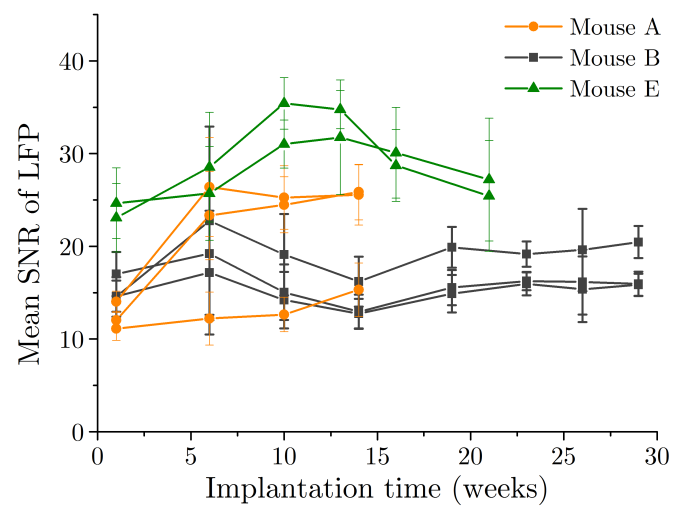

Figure 6: Evolution over time of the mean signal quality of the Local Field Potentials recorded from mice hippocampi by Parylene probes. Only the electrodes in working conditions (case 1) in the three mice that were implanted for longer periods is represented.

The results show that the SNR is high enough (between 11 and 35) for proper LFP hippocampal recordings. Besides, it seems that for a functional electrode, the SNR does not drop over the course of the trial, and remains at a stable value around 18. Fittingly, this demonstrate the feasibility to perform quality recordings over time with a Parylene implant. Interestingly, no significant difference between the performance of gold and PEDOT-modified electrodes was observed. That is, the oscillations measured in this trial are high enough so that even the gold electrodes quality enables us to record high quality signals. 
However, in our previous studies, PEDOT-nanostructured electrodes, while reducing electrode impedance by two orders of magnitude at $1 \mathrm{kHz}$, demonstrated real improvements for spontaneous single unit activity exhibiting bursts of action potentials [26]. Therefore future in vivo work should involve spike recording and sorting, so that SNR data and proper comparison between a gold and a PEDOT-nanostructured electrode can be gathered.

Besides, signal quality does not only come from device stability but also from body reaction to the implanted device. The modification of the surrounding environment plays a crucial part in electrode stability, and should definitely be assessed in the future.

\subsubsection{Morphology and impedance alteration}
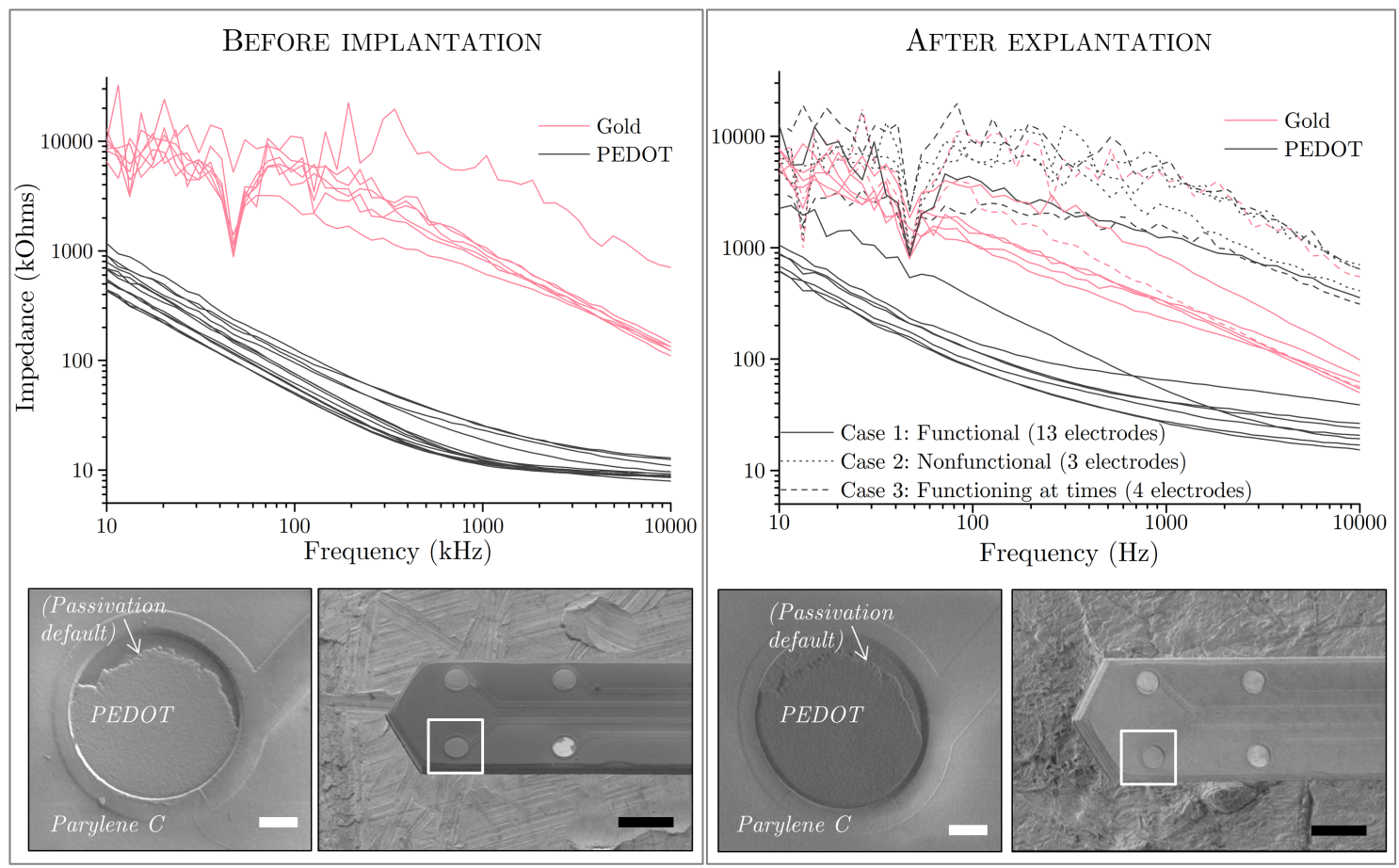

Figure 7: EIS evolution before implantation and after explantation ( 8 to 30 weeks) in mice brains for all the implanted electrodes, both gold (in pink) and PEDOT-nanostructured (in gray). In the EIS after explantation, electrode impedances are displayed depending on their level of functionality (functional, nonfunctional and functioning at times). All nonfunctional electrodes have indeed a very high impedance, as well as most electrodes that were functioning at times. This correlates the asumption that the unability to record was more likely to come from the device (packaging, faulty contact, mechanical damage) than from the foreign body response. Representative SEM images of a PEDOT-modified electrode and implant before and after in vivo 30 weeks implantation in a mouse hippocampus show no obvious sign of delamination nor swelling of the materials used for the fabrication (white scale bar is $10 \mu \mathrm{m}$, black scale bar is $100 \mu \mathrm{m}$ ). The absence of silk on the backside is consistent with its bioresorption in the time scale expected.

Finally, the alteration of the Parylene devices in vivo was assessed via EIS measurement in saline and SEM images, before implantation and after explantation (Figure 7). The SEM images comparison shows little biofouling effects, with no apparent sign of Parylene delamination, consistent with the in vitro results and the SNR measurements. Gold therefore PEDOT-modified electrodes present with a flaw both before implantation and after explantation, due to improper etching of the passivation layer on the wafer periphery. Although this defect is to be regretted, these images show that this weakness point had not propagated over time, and the PEDOT nanostructuration does not seem to be delaminating over time.

Impedance measurement after explantation can compared to whether the electrode was in funtioning state presented in the previous section. Both gold (in pink) and PEDOT-nanostructured (in gray) electrodes that were functioning throughout the trial (Case 1) presented with an impedance increase of roughly a factor of $2-3$ at $1 \mathrm{kHz}$ after explantation, except for one electrode whose impedance increased 
considerably. This degradation of electrode properties can stem from device material response in this biological environment over time, but also from biofouling effects, or even from the mechanical trauma experienced by the probe as it is removed from the mouse brain. On the other hand, all nonfunctional electrodes (Case 2) and most electrodes that were functioning at times (Case 3) have an impedance over $1 \mathrm{M} \Omega$ at $1 \mathrm{kHz}$, which correlates the assumption that the inability to record is more likely to come from the device (packaging, faulty contact, mechanical damage) than from the foreign body response.

On the other hand, it is noteworthy to underline the fact that no trace of silk coating was present on the retracted shank, even for the devices only implanted during 7 weeks. This suggests that all had indeed at least dissolved in the tissues, if not resorbed entirely, in agreement with theoretical degradation rate over a few days $[28,26]$.

\subsection{Conclusion}

Soft biomaterials such as Parylene $\mathrm{C}$ are increasingly studied for their use in biomedical implants, and more particularly intracortical devices. Their compliance is meant to alleviate the stress at the implant/tissue interface, therefore increasing implant acceptability in the long run, but the fate of these polymeric devices over time remains little known. Physiological conditions tend to impair immersed materials, through mechanical, chemical and electrical modifications. Swelling and delamination, among others, have shown to provoke device failure over time. In this work, we presented a preliminary assessment of the stability of Parylene C-based neural sensors for in vivo implantation. These $25 \mu \mathrm{m}$-thick Parylene implants each contain four recording gold electrodes nanostructured via electrochemical route using conductive polymer PEDOT to increase electrical capability. Both in vitro soaking trial in Artificial Cerebro-Spinal Fluid (ACSF) at $37^{\circ} \mathrm{C}$ and in vivo implantation in 5 mice brains were carried out for a period of 6 months, during which both morphology and electrical properties were monitored.

One of the major limitations encountered for both soaking and implantation test resides in the packaging and connectic reliability. Indeed, one out of four soaked implants showed early signs of water uptake in the connectic, while $30 \%$ of all electrodes implanted in vivo had partially or permanent faulty contacts. Connectic issues have often been listed as a predominant failure mode and should be especially optimized in the future. Apart from this matter, the Parylene devices seemed to withstand relatively well physiological conditions. The electrodes on the other three out of four implants all had stable impedance after six months in ACSF, that is within $10 \%$ of their initial value, and no significant morphological alteration was observed, neither of the PEDOT nanostructuration nor of the Parylene itself. This finding correlates with the in vivo test, where all electrodes that did not present with packaging issue, were able to accurately record wirelessly characteristic LFP of these mice discovering an unfamiliar environment, with steady Signal-to-Noise Ratio over the six months trial. In this experiment, no significant difference between gold and PEDOT electrodes recording capability was observed. EIS comparison before and after implantation showed an increase in impedance of a ratio around 2-3 for both electrode types at $1 \mathrm{kHz}$, while morphology assessment revealed little microscopic effect on the material. Once again, no delamination, swelling or consequent biofouling was observed on either soaked or implanted devices.

Future experiments should involve the recording and sorting of neural spikes in order to better characterize signal quality evolution over time. This would also enable us to better compare gold electrodes versus PEDOT-modified electrodes, and their relative expectancy over time. Finally, it is well-known that device biostability stems from both material reaction to its environment, but also from tissue response to the implanted device as a function of time. Therefore, immunohistochemical analysis of the biological response constitutes an obvious next step in line towards a more complete biostability assessment of Parylene-based neural implants.

\section{Acknowledgments}

The authors would like to thank Nanomade Concept for the use of the bonding equipment. This work was supported by LAAS-CNRS micro and nano technologies platform, a member of the french Renatech network. Part of this work was financed by ANR Neuromeddle 15-CE19-0006. The PhD grant was allocated by CNRS-INSIS. 


\section{References}

[1] S. Sommakia, H. C. Lee, J. Gaire, and K. J. Otto, "Materials approaches for modulating neural tissue responses to implanted microelectrodes through mechanical and biochemical means," Current Opinion in Solid State and Materials Science 18, pp. 319-328, Sept. 2014.

[2] R. L. Rennaker, J. Miller, H. Tang, and D. A. Wilson, "Minocycline increases quality and longevity of chronic neural recordings," Journal of neural engineering 4(2), pp. 1-5, 2007.

[3] J. K. Nguyen, D. J. Park, J. L. Skousen, A. E. Hess-Dunning, D. J. Tyler, S. J. Rowan, C. Weder, and J. R. Capadona, "Mechanically-compliant intracortical implants reduce the neuroinflammatory response," Journal of neural engineering 11, p. 056014, Oct. 2014.

[4] Z. Fekete and A. Pongrácz, "Multifunctional soft implants to monitor and control neural activity in the central and peripheral nervous system: A review," Sensors and Actuators B: Chemical 243(December), pp. 1214-1223, 2017.

[5] A. Hogg, S. Uhl, F. Feuvrier, Y. Girardet, B. Graf, T. Aellen, H. Keppner, Y. Tardy, and J. Burger, "Protective multilayer packaging for long-term implantable medical devices," Surface and Coatings Technology 255, pp. 124-129, 2014.

[6] H. W. Lo and Y. C. Tai, "Characterization of parylene as a water barrier via buried-in pentacene moisture sensors for soaking tests," International Conference on Nano/Micro Engineered and Molecular Systems , pp. 872-875, 2007.

[7] D. C. Rodger, A. J. Fong, W. Li, H. Ameri, A. K. Ahuja, C. Gutierrez, I. Lavrov, H. Zhong, P. R. Menon, E. Meng, J. W. Burdick, R. R. Roy, V. R. Edgerton, J. D. Weiland, M. S. Humayun, and Y. C. Tai, "Flexible parylene-based multielectrode array technology for high-density neural stimulation and recording," Sensors and Actuators, B: Chemical 132, pp. 449-460, June 2008.

[8] E. Meng, P.-Y. Li, and Y.-C. Tai, "Plasma removal of Parylene C," Journal of Micromechanics and Microengineering 18, p. 045004, Apr. 2008.

[9] A. Lecomte, A. Degache, E. Descamps, L. Dahan, and C. Bergaud, "Biostability Assessment of Flexible Parylene C-based Implantable Sensor in Wireless Chronic Neural Recording," Procedia Engineering 168, pp. 189-192, 2016.

[10] J. Black, Biological Performance of Materials: Fundamentals of Biocompatibility, marcel dek ed., 2006.

[11] C. D. Lee, S. A. Hara, L. Yu, J. T. W. Kuo, B. J. Kim, T. Hoang, V. Pikov, and E. Meng, "Matrigel coatings for Parylene sheath neural probes," Journal of Biomedical Materials Research Part B: Applied Biomaterials 104(2), pp. 357-368, 2015.

[12] S. A. Hara, B. J. Kim, J. T. W. Kuo, C. D. Lee, E. Meng, and V. Pikov, "Long-term stability of intracortical recordings using perforated and arrayed Parylene sheath electrodes," Journal of Neural Engineering 13(6), p. 066020, 2016.

[13] P. Moshayedi, G. Ng, J. C. F. Kwok, G. S. H. Yeo, C. E. Bryant, J. W. Fawcett, K. Franze, and J. Guck, "The relationship between glial cell mechanosensitivity and foreign body reactions in the central nervous system," Biomaterials 35(13), pp. 3919-3925, 2014.

[14] J. M. Anderson and Q. H. Zhao, "Biostability of biomedical polymers," MRS Bulletin, pp. 75-77, 1991.

[15] M. Jorfi, J. L. Skousen, C. Weder, and J. R. Capadona, "Progress towards biocompatible intracortical microelectrodes for neural interfacing applications," Journal of Neural Engineering 12(1), p. 011001, 2015. 
[16] C. Hassler, R. P. von Metzen, P. Ruther, and T. Stieglitz, "Characterization of Parylene C as an encapsulation material for implanted neural prostheses," Journal of biomedical materials research. Part B, Applied biomaterials 93, pp. 266-274, Apr. 2010.

[17] S. Minnikanti, G. Diao, J. J. Pancrazio, X. Xie, L. Rieth, F. Solzbacher, and N. Peixoto, "Lifetime assessment of atomic-layer-deposited Al2O3-Parylene C bilayer coating for neural interfaces using accelerated age testing and electrochemical characterization," Acta biomaterialia 10, pp. 960-967, Feb. 2014.

[18] J. P. Seymour, Y. M. Elkasabi, H. Y. Chen, J. Lahann, and D. R. Kipke, "The insulation performance of reactive parylene films in implantable electronic devices," Biomaterials 30(31), pp. 6158-6167, 2009.

[19] J. Agorelius, F. Tsanakalis, A. Friberg, P. T. Thorbergsson, L. M. E. Pettersson, and J. Schouenborg, "An array of highly flexible electrodes with a tailored configuration locked by gelatin during implantation : initial evaluation in cortex cerebri of awake rats," Frontiers in Neuroscience $\mathbf{9}$ (September), pp. 1-12, 2015.

[20] F. Wu, L. Tien, F. Chen, D. Kaplan, J. Berke, and E. Yoon, "A multi-shank silk-backed parylene neural probe for reliable chronic recording," 2013 Transducers and Eurosensors (June), pp. 888-891, 2013.

[21] B. J. Kim, J. T. W. Kuo, S. A. Hara, C. D. Lee, L. Yu, C. A. Gutierrez, T. Q. Hoang, V. Pikov, and E. Meng, "3D Parylene sheath neural probe for chronic recordings," Journal of Neural Engineering 10(4), p. 45002, 2013.

[22] V. Castagnola, C. Bayon, E. Descamps, and C. Bergaud, "Morphology and conductivity of PEDOT layers produced by different electrochemical routes," Synthetic Metals 189, pp. 7-16, Mar. 2014.

[23] M. R. Abidian, L. G. Salas, A. Yazdan-Shahmorad, T. C. Marzullo, D. C. Martin, and D. R. Kipke, "In-vivo evaluation of chronically implanted neural microelectrode arrays modified with poly $(3,4-$ ethylenedioxythiophene) nanotubes," Proceedings of the 3rd International IEEE EMBS Conference on Neural Engineering, pp. 61-64, 2007.

[24] K. A. Ludwig, J. D. Uram, J. Yang, D. C. Martin, and D. R. Kipke, "Chronic neural recordings using silicon microelectrode arrays electrochemically deposited with a poly(3,4-ethylenedioxythiophene) (PEDOT) film," Journal of neural engineering 3(1), pp. 59-70, 2006.

[25] V. Castagnola, E. Descamps, A. Lecestre, L. Dahan, J. Remaud, L. Nowak, and C. Bergaud, "Parylene-based flexible neural probes with PEDOT coated surface for brain stimulation and recording," in Biosensors and Bioelectronics, 67, pp. 450-457, Elsevier, Sept. 2014.

[26] A. Lecomte, V. Castagnola, E. Descamps, L. Dahan, M. Blatché, E. Leclerc, and C. Bergaud, "Silk and PEG as means to stiffen a parylene probe for insertion in the brain: toward a double time-scale tool for local drug delivery," Journal of Micromechanics and Microengineering 25(12), p. 125003, 2015.

[27] A. Lecomte, A. Lecestre, D. Bourrier, M.-c. Blatché, E. Descamps, and C. Bergaud, "Deep plasma etching of Parylene C patterns for biomedical applications," Microelectronic Engineering 177, pp. 70$73,2017$.

[28] H.-J. Jin, J. Park, V. Karageorgiou, U.-J. Kim, R. Valluzzi, P. Cebe, and D. L. Kaplan, "Waterstable silk films with reduced $\beta$-sheet content," Advanced Functional Materials 15, pp. 1241-1247, Aug. 2005.

[29] C. Zheng, K. W. Bieri, Y. T. Hsiao, and L. L. Colgin, "Spatial Sequence Coding Differs during Slow and Fast Gamma Rhythms in the Hippocampus," Neuron, pp. 1-11, 2015.

[30] J. E. Lisman and O. Jensen, "The Theta-Gamma Neural Code," Neuron 77(6), pp. 1002-1016, 2013. 
[31] S. Suner, M. R. Fellows, C. Vargas-Irwin, G. K. Nakata, and J. P. Donoghue, "Reliability of signals from a chronically implanted, silicon-based electrode array in non-human primate primary motor cortex," IEEE Transactions on Neural Systems and Rehabilitation Engineering 13(4), pp. 524-541, 2005.

[32] M. P. Ward, P. Rajdev, C. Ellison, and P. P. Irazoqui, "Toward a comparison of microelectrodes for acute and chronic recordings," Brain Research 1282, pp. 183-200, 2009. 


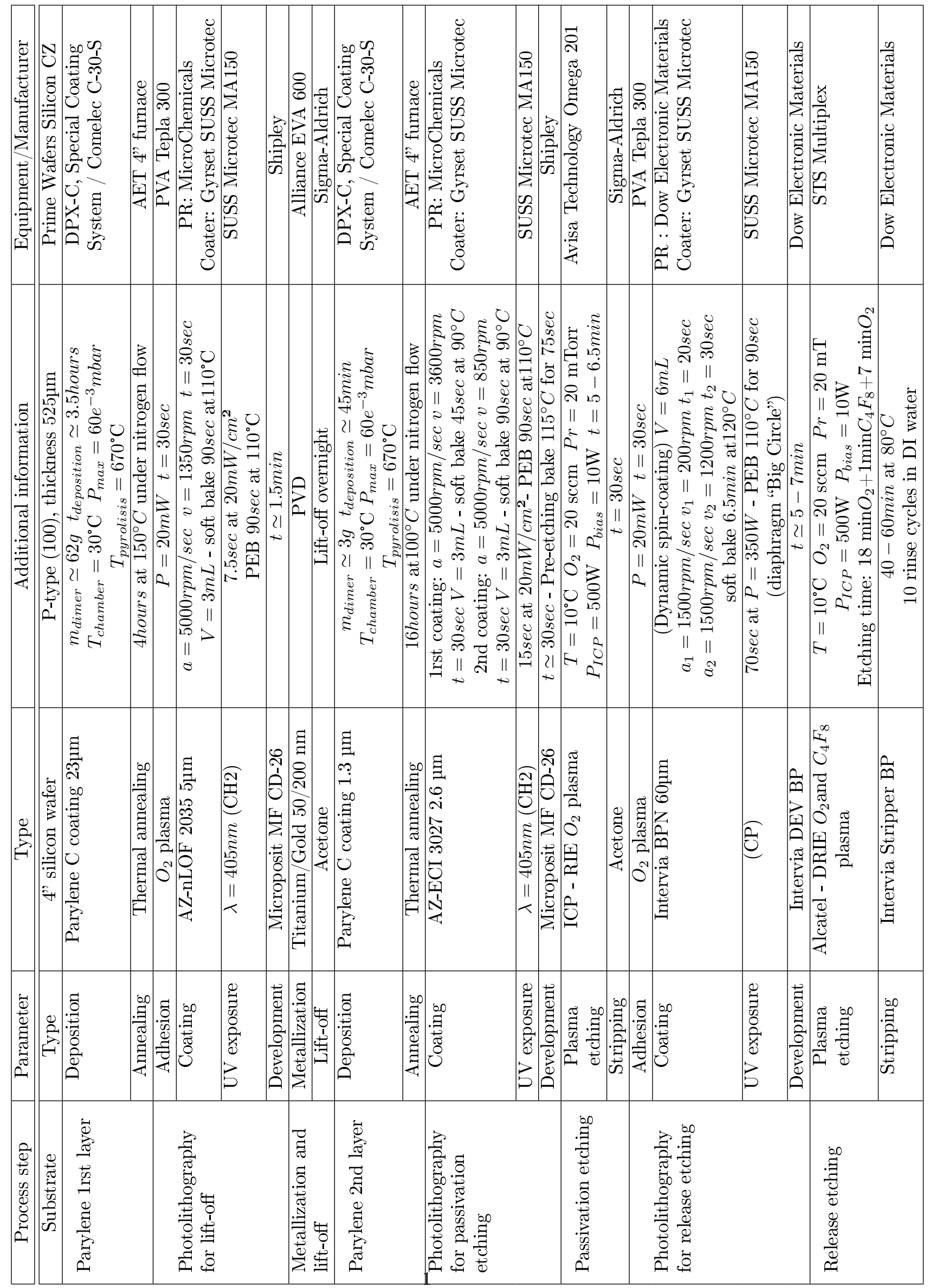

Supplementary data

Parylene neural probe microfabrication process, parameters and commercial references. 


\section{Supplementary data}

(a) Impedance and (b) phase of the four PEDOT-modified electrodes (a,b,c and d) present on a Parylene C probe. Measurements are carried out in either a $25 \mathrm{~mL}$ solution of Artificial CerebroSpinal Fluid (ACSF, solid line) or $\mathrm{NaCl} 0.9 \mathrm{w} / \mathrm{v}$ (dash line) at room temperature. The difference between EIS measurement in $\mathrm{NaCl}$ or in ACSF solutions is in the order of magnitude of EIS variability measurement. Impedance in ACSF is slightly higher than in $\mathrm{NaCl}$ because ACSF contains many other ions (see Table for comparison) but only $0.725 \mathrm{w} / \mathrm{v}$ $\mathrm{NaCl}$, which is the main component of ion current.
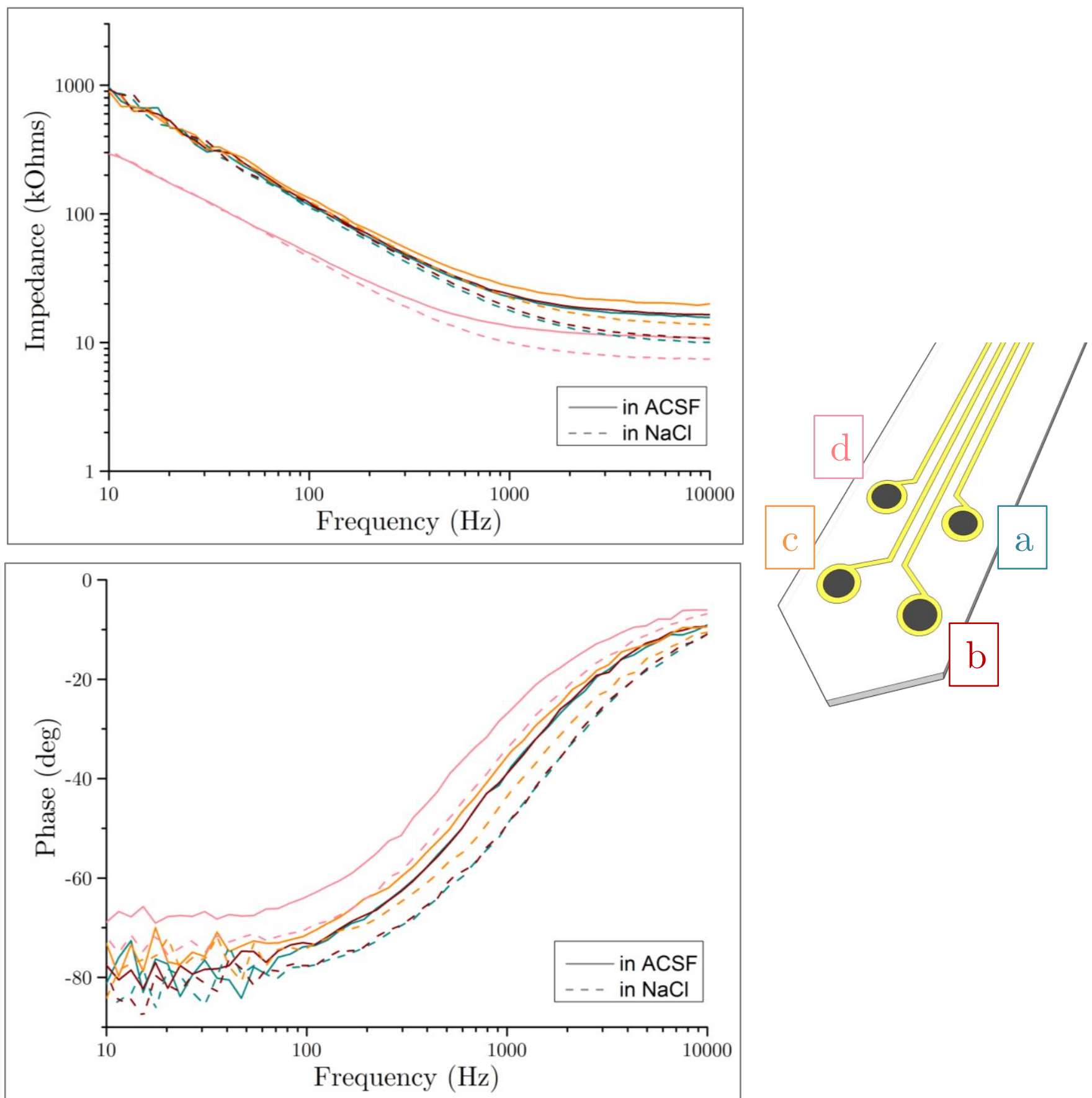